\title{
Magnetic Resonance Imaging of Neuronal Ceroid Lipofuscinosis in a Border Collie
}

\author{
Hiroshi KOIE ${ }^{1)}$, Hisashi SHIBUYA ${ }^{2)}$, Tsuneo SATO'), Akane SATO $^{2)}$, Koji NAWA ${ }^{3)}$, Yuko NAWA ${ }^{3)}$, \\ Masato KITAGAWA ${ }^{4}$, Manabu SAKAI ${ }^{1)}$, Tomoko TAKAHASHI ${ }^{1)}$, Yoshiki YAMAYA ${ }^{1)}$, Osamu YAMATO ${ }^{5}$, \\ Toshihiro WATARI ${ }^{1)}$ and Mikihiko TOKURIKI ${ }^{1)}$ \\ ${ }^{1)}$ Laboratories of Comprehensive Veterinary Clinical Studies, ${ }^{2)}$ Veterinary Pathology and ${ }^{4)}$ Veterinary Preventive Medicine and Animal \\ Health, Department of Veterinary Medicine, College of Bioresouce Sciences, Nihon University, 1866 Kameino, Fujisawa, Kanagawa 252- \\ 8510, ${ }^{3)}$ Minamihara Pet Clinic, 1-4-24 Minamihara, Yamagata 990-2413 and ${ }^{5)}$ Laboratory of Internal Medicine, Department of \\ Veterinary Clinical Sciences, Graduate School of Veterinary Medicine, Hokkaido University, Sapporo 060-0818, Japan
}

(Received 5 March 2004/Accepted 2 June 2004)

ABSTRACT. A castrated male border collie 23 months of age weighing $19.4 \mathrm{~kg}$ was referred to the Animal Medical Center of Nihon University with complaints of visual disturbance and behavioral abnormality, hyperacusis and morbid fear. The MRI examination revealed the slight dilated cerebral sulci and cerebellar fissures and left ventricular enlargement. This is the first report of MRI fin dings of canine neuronal ceroid lipofuscinosis.

KEY WORDS: border collie, MRI, neuronal ceroid lipofuscinosis.

J. Vet. Med. Sci. 66(11): 1453-1456, 2004

Neuronal ceroid lipofuscinosis (NCL) belongs to a group of inherited lysosomal storage diseases characterized by the deposition of autofluorescent lipopigments in neurons and other cells. In small animal neurology, canine NCL has been classified on clinical grounds, especially the age of onset, as prepubertal-protracted, early adult acute or adult onset but, the dog breed may be genetically of importance for its classification, because the mutation is more likely to arise from a common progenitor [3,6]. Extensive studies on canine NCL had been reported in English setter dogs whose disorder shared similarities with human juvenile NCL. It had been long considered to be a good animal model for human juvenile NCL until defects in the canine ortholog of the CLN 3 gene were ruled out as the cause of the disease in this breed [8]. NCL cases in border collies have been reported and the pedigree analysis allowed the carriers to predict the possibility of NCL in some families [9, 10]. This report describes the clinical, MRI, biochemical, microscopic, and ultrastructural features of ceroid lipofuscinosis in a border collie.

A castrated male border collie 23 months of age weighing $19.4 \mathrm{~kg}$ was referred to Nihon University Animal Medical Center with complaints of visual disturbance and behavioral abnormality as hyperacusis, morbid fear and less responsive to verbal commands. Clinically, there were no noticeable changes in the blood test, neurological examination and analysis of the cerebrospinal fluid. The examination of the optical fundus and electroretinogram could not detect any abnormalities. There was also no indication of canine distemper infection, because the serum distemper antibody titer was 1280 -fold and the serum neutralizing antibody titer was 550-fold; the distemper antibody titer of the cerebrospinal fluid was less than ten-fold and the neutralizing antibody titer was three-fold but, the dog's condition had deteriorated gradually since forty days after the first examination, showing neurological symptoms such as ataxia and hypermetria, incontinence, and difficulty in prehending and chewing food. Ninety-five days after first presentation, the dog had diarrhea with hemorrhage and hyperthermia of $42^{\circ} \mathrm{C}$, and the next day died with convulsion.

On the day of the first medical examination, the brain was evaluated by MRI with a 0.5 tesla superconducting MRI system (FREXART MRT-50GP, Toshiba Medical Systems Co., Tokyo, Japan) set at TR/TE $=350 / 15$ milliseconds in a T1-weighted image, TR/TE $=4000 / 120$ milliseconds in a T2weighted image and $\mathrm{TR} / \mathrm{TE}=8000 / 120$ milliseconds in a FLARE image with 4-mm-thick slices.

The cerebrum and the eyeballs were dissected on the day of death, but further necropsy was not carried out at the owner's request. The tissues were fixed in $10 \%$ formalin for histological study and in a mixture of $1 \%$ glutaraldehyde and $4 \%$ formalin solution for electron microscopic examination. Paraffin-embedded sections were prepared and stained with hematoxylin and eosin (H\&E), luxol fast blue with H\&E (LFB-HE), PAS and schmorl. Frozen sections were prepared at a thickness of $10 \mu \mathrm{m}$ on a cryostat and stained with sudan III. A fluorescence microscope (Olympus BH2RFC, Tokyo) was employed for observation of the autofluorescent granules. Fluorescent emission from a mercury vapor lamp was passed through an excitation filter which consisted of a 405, 435 and $490 \mathrm{~nm}$ bandpass. For electron microscopy, a fresh sample was diced, and fixed in $1 \%$ osmium tetroxide with $0.1 \mathrm{M}$ cacodylic acid buffer. After being embedded in epoxy resin, ultrathin sections were prepared, stained with uranyl acetate and lead citrate and examined with an electron microscope (JEM 1200 EX).

The pedigree for the present case is shown in Fig.1. One female dog (V-2 in Fig. 1) of the litter five followed the same course as the present case (V-1) and finally died. This female case was not pathologically diagnosed. Dogs IV-1 and IV-2 were not affected. The present case was proven to be NCL by the pathologic examination. Therefore, it was 


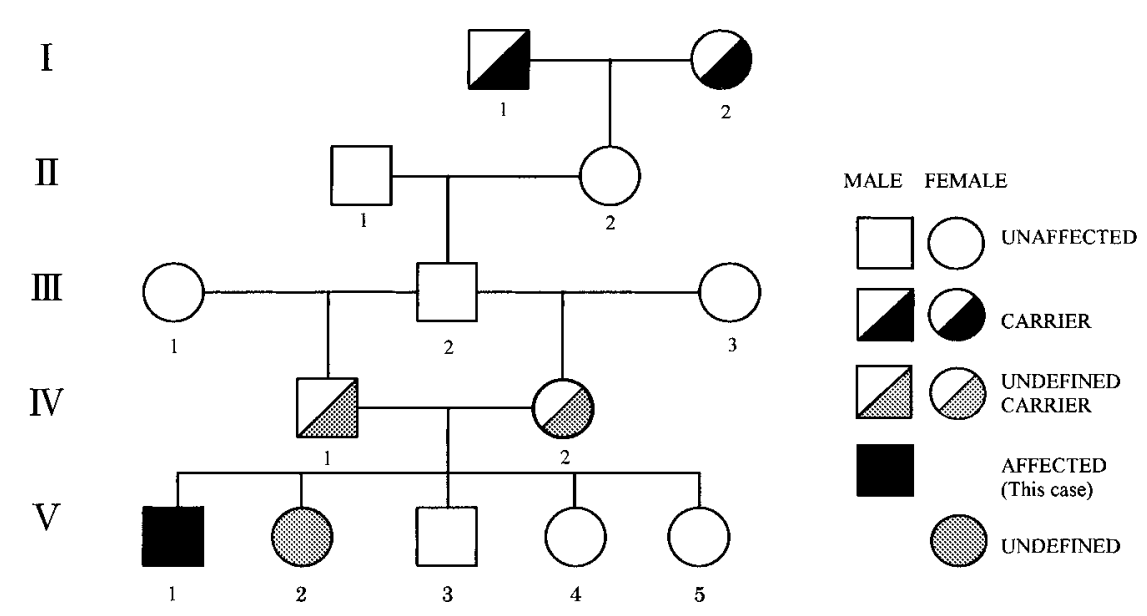

Fig. 1. Pedigrees of the affected dogs. The parents of the probands were not affected. V-2 followed the same course and finally died. V-2 did not have a pathological diagnosis made. IV-1 and IV-2 had the same sire (III-2) and I-1 and I-2 are officially recognized as carriers with the Border Collie Club of New South Wales Inc., Australia.

suspected that IV-1 and IV-2 were carriers in accordance with recessive autosomal gene inheritance based on Mendel's laws. When the pedigree was traced, IV-1 and IV-2 had the same sire, and dogs I-1 and I-2 are officially recognized as a carrier by the Border Collie Club of New South Wales Inc., Australia (Fig. 1).

The MRI examination revealed slight dilated cerebral sulci, left ventricular enlargement (Fig. 2) and dilation of cerebellar fissures (Fig. 3).

The microscopic examination revealed slight atrophy of the cerebral cortex with gliosis and a reduction in the number of neurons. H \& E stain showed that most neurons and glial cells contained eosinophilic cytoplasmic pigments. Their granules were irregular, slightly refractile and occasionally condensed. LFB, PAS, schmorl and Sudan III stains showed that the pigments were autofluorescent and positive (Fig. 4). In the retina, the ganglion cells were decreased in number and contained pigmented storage granules. The structure of the retina was well preserved.

Ultrastructural examination revealed that the storage granules varied in size from $300 \mathrm{~nm}$ to $4 \mu \mathrm{m}$, and formed such shapes as fingerprint-like, curvilinear, amorphous, membranous or mixed patterns (Fig. 5). Most granules had no distinct limited membrane.

The NCL of border collies was first recognized in Australia and New Zealand [10]. In 1992, the NCL of a male border collie of unknown pedigree was reported in the United States [5]. There is one NCL case of a chihuahua with hydrocephalus in Japan [7], but the genetic background is not clarified in the report. Our case became the first report that proved inherited NCL in Japan and the first case clinically investigated by MRI in a dog with NCL. It had been reported that CT showed dilatation of the ventricles, mildly widened cerebral sulci, and atrophy of the cerebral cortex in canine NCL [5]. Nuclear magnetic resonance (NMR) find- ings were documented. The NMR images could not demonstrate any clear-cut of brain damage [1]. MRI seems to be much more useful than CT examination for cranial disease, as many MRI diagnoses of human NCL had been reported. The MRI findings of human NCL included the dilation of the cerebral sulcus and ventricular enlargement [4], as well as defects including mild to moderate cerebellar atrophy in older cases [2]. It was reported that thalamic hypointensity, high-signal rims around the entire lateral ventricles, and white matter hyperintensity are evident on T2-weighted images in human ceroid lipofuscinosis [11]. Our case also showed dilation of the cerebral sulci, ventricular enlargement and dilation of the cerebellar fissures with MRI. In a histological study, the cerebral cortex was slightly atrophied, with gliosis and a reduction in the number of neurons. Therefore, it may be suggested that abnormal images revealed with MRI including dilation of cerebral sulci, cerebellar fissures and ventricular enlargement were caused by brain atrophy. In our case, there were no thalamic hypointensity, high-signal rims around the entire lateral ventricles, or white matter hyperintensity on T2-weighted images. As MRI reflects pathological change, it could serve as an antemortem diagnosis for canine NCL. If we are able to collect a lot of NCL cases, it may be possible to establish the imaging diagnosis, especially MRI features and differential diagnosis of NCL.

\section{REFERENCES}

1. Armstrong, D., Quisling, R. G., Webb, A. and Koppang, N. 1983. Neurobiol. Aging. 4: 297-303.

2. Autti, T., Raininko, R., Vanhanen, S. L. and Santavuori, P. 1994. Neuroradiology. 38: 476-482.

3. Cantile, C., Buonaccorsi, A., Pepe, V. and Arispici, M. 1996. Prog. Vet. Neurol. 7: 82-87.

4. D'Incerti, L. 2000. Neurol. Sci. 21: S71-S73. 

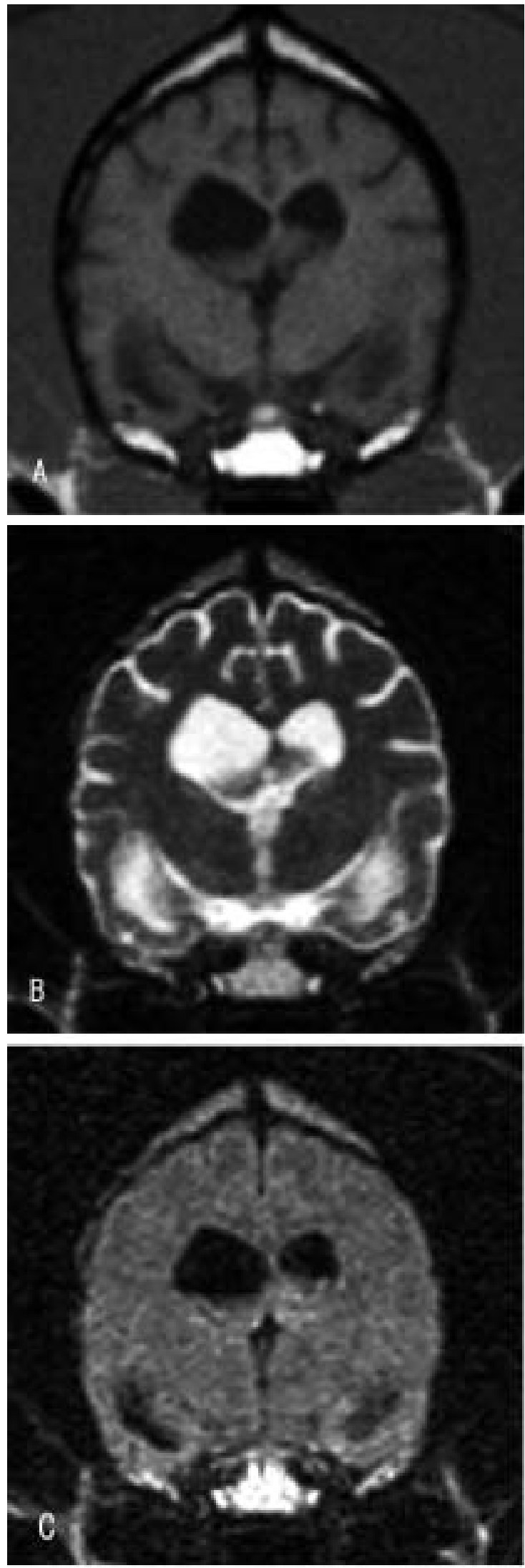
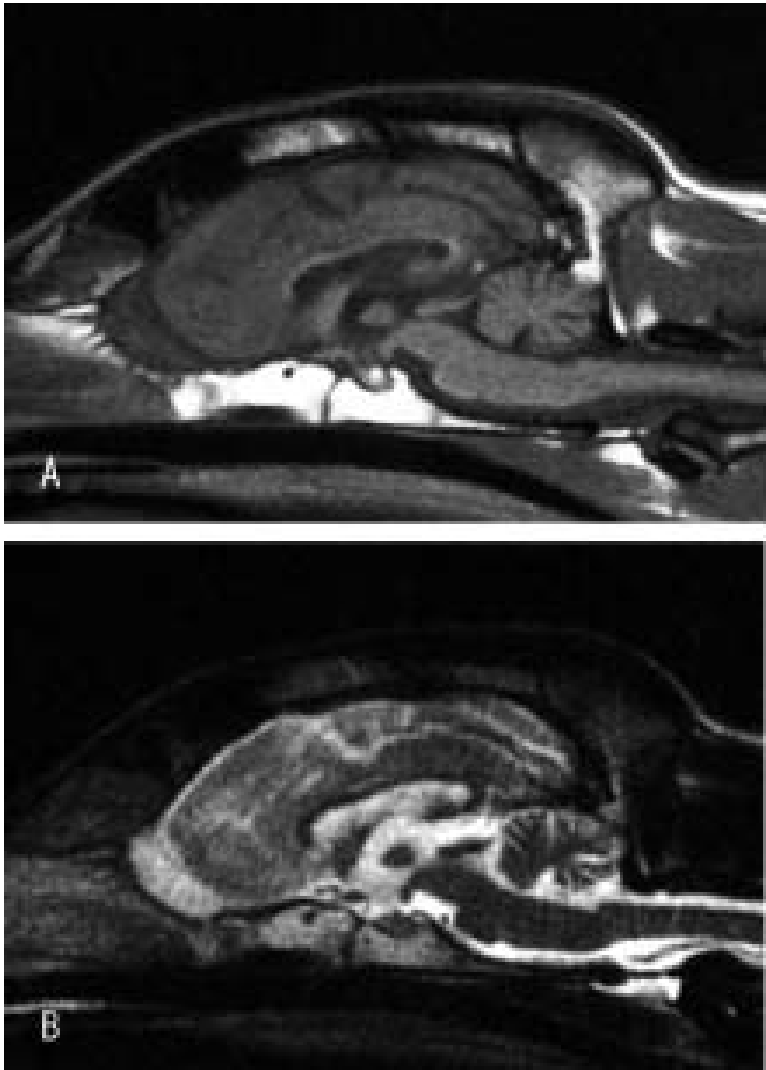

Fig. 3. $\mathrm{T}_{1}$ (A) and $\mathrm{T}_{2}$ (B) weighted sagittal section, which shows dilation of the cerebellar fissures. $\mathrm{T}_{1}: \mathrm{TR} / \mathrm{TE}=350 / 15$ milliseconds, $\mathrm{T}_{2}$ : TR/TE=4000/120 milliseconds with 4-mmthick slices.

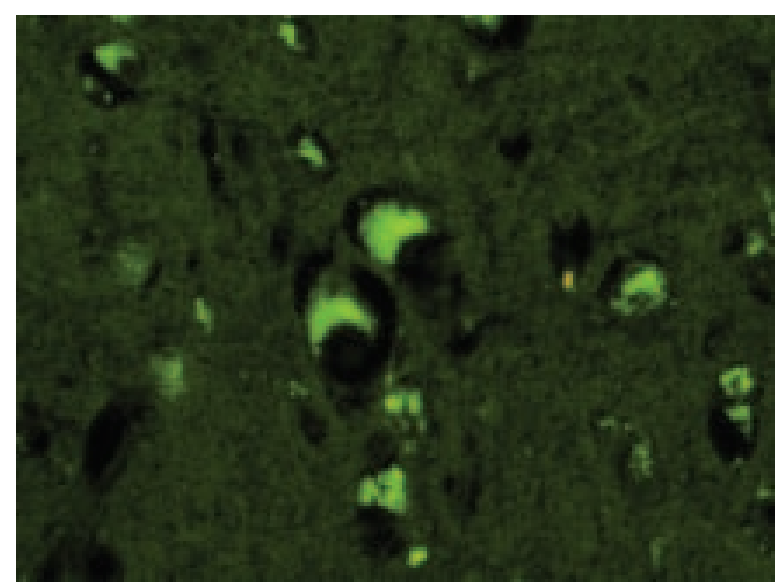

Fig. 4. Most neurons and glia cells in the cerebral cortex contain the autofluorescent storage granules $(\times 100)$.

Fig. 2. $T_{1}$ (A) and $T_{2}$ (B) weighted, FLARE (C) Transverse image which shows dilation of the cerebral sulci and left ventricular enlargement. $\mathrm{T}_{1}: \mathrm{TR} / \mathrm{TE}=350 / 15$ milliseconds, $\mathrm{T}_{2}: \mathrm{TR} /$ $\mathrm{TE}=4000 / 120$ milliseconds, FLARE: TR/TE $=8000 / 120$ milliseconds with 4-mm-thick slices. 


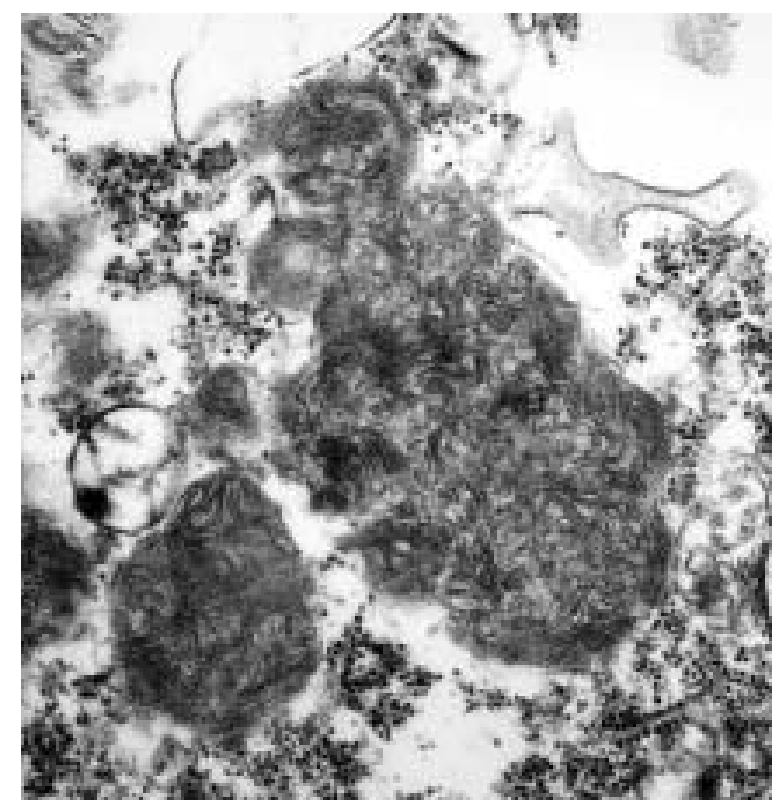

Fig. 5. Electron micrograph of the storage granules which varied in size and pattern and formed shapes such as fingerprintlike, curvilinear, amorphous, membranous and mixed $(\times$ $75,000)$.
5. Franks, J. N., Dewey, C. W., Walker, M. A. and Storts, R. W. 1999. J. Am. Anim. Hosp. Assoc. 35: 430-435.

6. Jolly, R.D., Palmer, D. N., Studdert, V. P. Sutton, R. H., Kelly, W. R., Koppang, N., Dahme, G., Hartley, W. J., Patterson, J. S. and Riis, R.C. 1994. J. Small Anim. Prac. 35: 299-306.

7. Kuwamura, M., Hattori, R., Yamate, J., Kotani, T. and Sasai, K. 2003. J. Small Anim. Prac. 44: 227-230.

8. Shibuya, H., Liu, P. C., Katz, M. L., Siakotos, A. N., Nonneman, D. J. and Johnson, G. S. 1998. J. Neurosci. Res. 52: 268275.

9. Studdert, V. P. and Mitten, R. W. 1991. Aust. Vet. J. 68: 137140.

10. Taylor, R. M. and Farrow, B. R. H. 1988. Acta Neuropathol. 75: 627-631.

11. Vanhanen, S.L., Raininko, R., Autti, T. and Santaruori, P. 1995. J. Child Neurol. 10: 444-450. 